\title{
Piezoelectric Based Wireless Charger
}

\author{
Rishi Sikka
}

\begin{abstract}
This paper proposes a piezoelectric based wireless charger. Human being is using various types of energy resources that comes to extent level. Due to which there is a need to develop a system that generate energy in a most economical manner which causes no harm to human beings and animals living on earth. This paper offers the implementation of piezoelectric sensor on the shoe of a person so that it generates electrical energy on the movement. Therefore, it can be said that the article illustrates the design and implementation of the energy collecting scheme by converting energy due to human movement into usable form so that it can charge any battery wirelessly.
\end{abstract}

Keywords- Piezoelectric sensor, energy resources, foot movement, wireless charger.

\section{INTRODUCTION}

Humans have been using energy at an ever-increasing rate for their existence and well-being since coming to world millions of years ago [1]. Interest in research and the development of advanced technology has been particularly fast in present ages [2]. As technology amplified, the problems also associated with it, such as the fast battery drainage. Now think that your phone is getting charged when you go. This can be done by Piezo electric sensor that charges a cell phone by electric power movement. The keys to this technique are piezoelectricity and Wireless power transfer (WTP). Energy generation from human movement is a way of finding clean and sustainable electricity [3], [4]. The electric energy produced from jumping, walking or running is due to the mechanical pressure. When the piezoelectric crystal is weighed, a negative cost is made in the extended side and a positive cost on the near side [5]. The current flows over the material, when the weight is relieved. The piezoelectric sensor has been using since it has a capability of generating electricity by applying force either by jumping on it or by walking on it [6]. In wireless energy distribution system, the electrical energy is transmitted from power source (piezoelectric power generator) to a storage of battery or recycling battery (such as any electrical appliances) without any physical attachment such as wires or directors [7],[8]. Therefore, the energy is harvested from human movements and is spread out quickly with the capability to move wireless power and is used to dose the mobile battery.

\section{PROPOSED SYSTEM \& RESULTS}

In piezoelectricity there are two effects: direct and indirect piezoelectric effect [9]. In a direct piezoelectric effect, the electrical generation occurs when a mechanical strain i.e. technical emphasis is placed on piezoelectric materials; but the effect of the crystal changed to the piezoelectric materials is the changed effect of it. The effect

Revised Manuscript Received on 14 September, 2019.

Rishi Sikka, Department of Electronics Engineering, Sanskriti University, Uttar Pradesh, India.(Email: sanpubip@gmail.com) of direct piezoelectric is used in the manufacture of production and the effects of piezoelectric are used in manufacturing producers. The materials of piezoelectric are both natural and voluntarily manufactured.

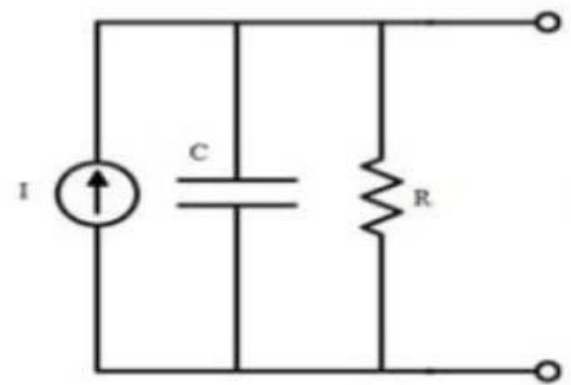

Fig.1 Circuit diagram of Piezo electric generator

$$
\begin{aligned}
& D=d \cdot T+T \cdot E \\
& S=s E \cdot T+d t . E
\end{aligned}
$$

The piezoelectric effect is represented by equation 3 and 4. Equation 3 represents the direct form of piezoelectric effect and eq.4 represents the converse form of piezo electric effect [10].

Table l. Energy storage density comparison

\begin{tabular}{|l|l|}
\hline $\begin{array}{l}\text { Energy harvester } \\
\text { Working }\end{array}$ & Energy Storage Density \\
\hline Principle & $(\mathrm{mJ} / \mathrm{cm} 3)$ \\
\hline & \\
\hline Electromagnetic & 24.8 \\
\hline Electrostatic & 4 \\
\hline Piezoelectric & 35.4 \\
\hline
\end{tabular}

There are three units in the design: an electric generation unit, a storing unit and a transmission unit. The storage side of the storing unit for Generation is out of the electricity generator Piezo, a bank capacitance / recharge battery (storing unit). This unit is in the shoe. Fig. 2 represent the design of the unit system. Transmitter-Receiver which contains a built-in bank, receives the electric signal from piezo and transmit to the storing unit and cell phone is charge throughout the movement of shoe. Moreover, a comparative measurement that shows the percentage of charge stored on new network assets is replenished.

Published By: Blue Eyes Intelligence Engineering 


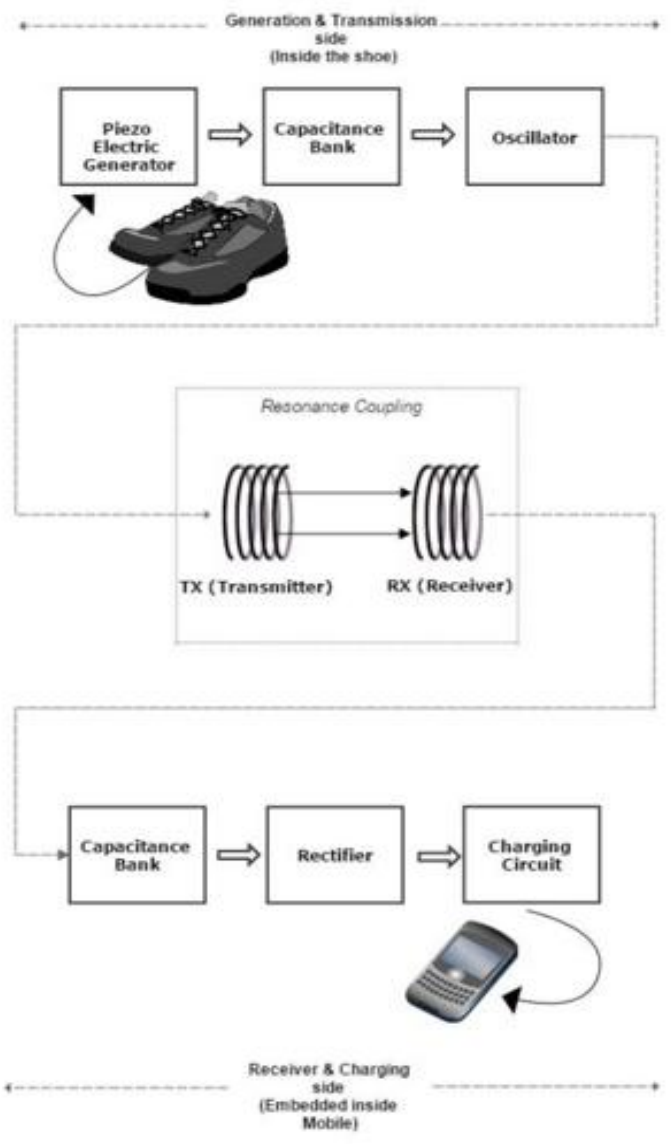

Fig.2. Block diagram of Piezo electric wireless power transfer mobile charging technique.

The working model of piezoelectric based wireless charger is shown in above figure.

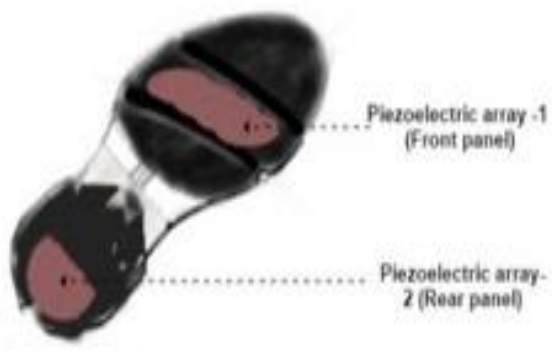

\section{Fig.3. Arrangement of piezoelectric sensors in the piezoelectric generator in the shoe sole.}

The wireless energy supply includes portable applications and non-portable use. They reduce installation costs by eliminating wire and this feature is particularly important where low energy sources are not available locally. In present era there is an increasing interest in exploring and developing wireless power technology to remove the "last" cable after Wi-Fi has been widely accepted [9]. The wireless electrical capacity used here is based on a strong current between electromagnetic corals to move power to a wireless between them [11]. Essentially the transmitter and receiver comprise of two coils; a transmitter's and a receiver's coil. The transmitter's coil is powered by the alternating current to make a magnetic field, which then affects a voltage in the receptor coil.

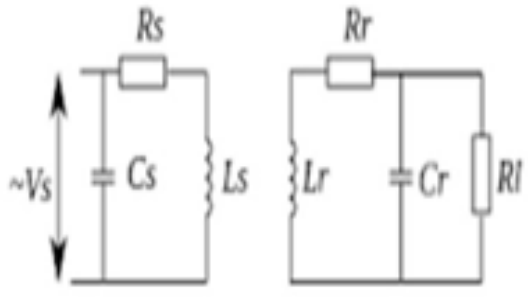

Fig 4. Inductive coupling

The transmission of energy takes place between two coils through fields of magnets. However, in this way, the distance between two coils should be minimal (a few $\mathrm{cm}$ ). The general access principle between two coils is used to flow electric energy without using wires [12]. The best demonstration of how reinstatement work can be done as the transformative, where there is no corporate communication between a primary coil and the secondary coils. Energy transfer will come forward due to the electromagnetic induction without physical connection between them.

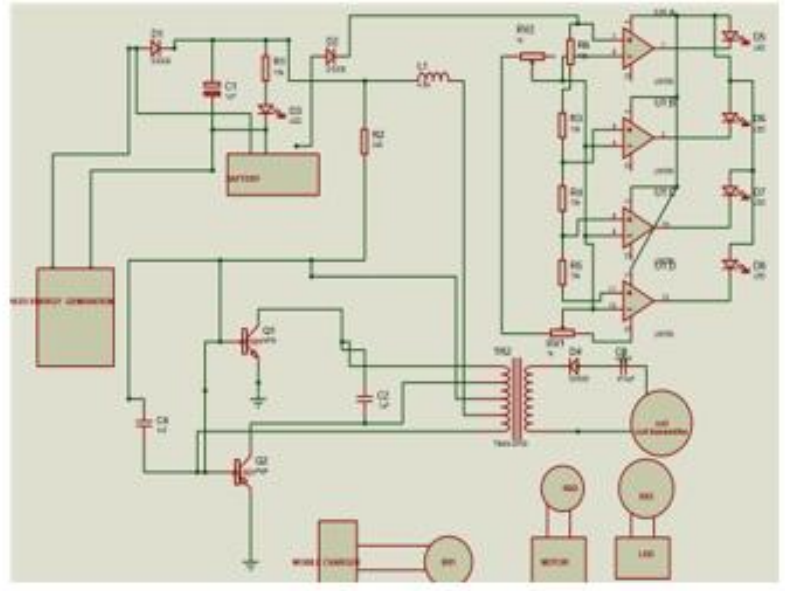

Fig 5: prototype circuit diagram

The AC power generated by the movement of shoe is then converted into DC. The bridge rectifier is used for converting pulsating ac power generated by piezo electric generator into dc power. Further for obtaining constant and smooth dc power, a filtration unit is connected to bridge rectifier so that smooth power is obtained. A filtration unit comprises of plurality of capacitors, resisters and inductors for doing filtering process. This power is filtered further by using filter elements. This power is governed by a regulator, and this power is then used for charging the battery of mobile or any other electrical equipment. The filtered power is received by RX unit and then send it to charge the battery remotely and wirelessly. An amplifying unit is used to boost up the power received by receiver coil and used for charging mobile battery. 


\section{CONCLUSION}

In this paper a wireless piezoelectric based mobile charger is designed for charging the battery of mobile phone wirelessly without the use of external power supply. In the proposed system, the piezoelectric sensor is put inside the heel or toe of the shoe pair. Both the piezoelectric generates electricity while walking, jumping or running. It can be said that the electricity is generated by piezo during the movement of the shoe wearer by the user. The user connects the mobile phone with the piezoelectric charger wirelessly. The main advantage of proposed system is that it does not cause any pollution and does not need any external supply to operate.

\section{REFERENCES}

1. International Renewable Energy Agency (IRENA), "Global Energy Transformation: A Roadmap to 2050," 2018.

2. The International Renewable Energy Agency, "Renewable Energy Integration in Power Grids. Technology Brief," 2015.

3. X. Li, Y. Yin, L. Fiondella, and Y. Zhou, "Software reliability analysis considering correlated component failures with coupling measurement framework," J. Syst. Eng. Electron., 2015.

4. D. Pradeep Kumar Reddy and J. Mohana, "Wireless power transfer,” Int. J. Pharm. Technol., 2016.

5. K. Uchino, Advanced piezoelectric materials. 2010.

6. M. Okayasu and K. Watanabe, "A study of the electric power generation properties of a lead zirconate titanate piezoelectric ceramic," Ceram. Int., 2016.

7. S. G. Kim, S. Priya, and I. Kanno, "Piezoelectric MEMS for energy harvesting," MRS Bulletin. 2012.

8. F. Yildiz, "Potential Ambient Energy-Harvesting Sources and Techniques," J. Technol. Stud., 2016.

9. H. D. Espinosa, R. A. Bernal, and M. Minary-Jolandan, "A review of mechanical and electromechanical properties of piezoelectric nanowires," Advanced Materials. 2012.

10. J. Yvonnet, "Piezoelectricity," in Solid Mechanics and its Applications, 2019.

11. A. J. Fleming and K. K. Leang, "Piezoelectric transducers," in Advances in Industrial Control, 2014.

12. C. De Marqui, "Piezoelectric energy harvesting," in Dynamics of Smart Systems and Structures: Concepts and Applications, 2016 